\title{
Can Software Transactional Memory make concurrent programs simple and safe?
}

\author{
Ketil Malde \\ Institute of Marine Research, Bergen, Norway \\ ketil.malde@imr.no
}

\begin{abstract}
Keywords: $\quad$ Software Transactional Memory:Genome Assembly
Abstract: $\quad$ Parallel programs are key to exploiting the performance of modern computers, but traditional facilities for synchronizing threads of execution are notoriously difficult to use correctly, especially for problems with a non-trivial structure. Software transactional memory is a different approach to managing the complexity of interacting threads. By eliminating locking, many of the complexities of concurrency is eliminated, and the resulting programs are composable, and thus simplifies refactoring and other modifications. Here, we investigate STM in the context of genome assembly, and demonstrate that a program using STM is able to successfully parallelize the genome scaffolding process with a near linear speedup.
\end{abstract}

\section{INTRODUCTION}

As multi-core processors are becoming commonplace, parallel programs are crucial for performance critical computation. Many problems can easily be partitioned into subproblems that can be solved independently (so-called "embarrassingly parallel" problems) but other problems are inherently more complicated, and are best solved by multiple interacting threads. In this case, care must be taken to keep separate threads of execution from interacting in ways that cause the program to behave incorrectly.

Traditionally, the shared data in parallel programs is protected by synchronization primitives (locks) that prevent simultaneous access to data structures. However, it is still quite difficult to write correct programs using these primitives, and incorrect or careless usage cause well-known problems like deadlocks and race conditions (Lee, 2006). In addition, independent program parts that use locking primitives are in general not composable, and for instance, refactoring a previously correct program can introduce new synchronization problems (Harris et al., 2005).

Software transactional memory (Shavit and Touitou, 1995), or STM, represents a different approach. Here, state that is shared between threads is accessed in transactions, and the state is stored in transactional variables. If multiple threads run simultaneous transactions that attempt to modify the same state, only one of the transactions succeeds, the others are rolled back and will be rescheduled by the run- time system.

Since there is no explicit locking, deadlocks are eliminated, and transactions are either committed completely or not at all, so intermediate (and possibly inconsistent) state is never exposed. In addition, STM transactions are composable (Harris et al., 2005). The disadvantage is a potentially higher overhead, both because transactions need to log access to transactional variables, and because transactions sometimes need to be restarted from scratch, which duplicates work.

Here, we investigate how STM can be applied to the problem of genome scaffolding, the process where the components of a partially assembled genome sequence are ordered and oriented to provide a more coherent (but often discontiguous) whole. A scaffolder program is implemented in Haskell using STM, and achieves a near linear speedup with the number of processors.

\subsection{Software Transactional Memory in Haskell}

There exist implementations of software transactional memory for many programming languages (e.g., Brevnov et al., 2008; Ni et al., 2008). Some of the problems faced by implementers is that the encapsulation of transactions is not easily enforced, and exceptions, I/O operations and global, mutable state can break the transaction abstraction. Harris et al. (2005) 
discuss this in more detail.

One distinguishing feature that sets Haskell apart from the majority of programming languages, is that it is pure: the result of a function depends only on its parameters, and the return value may not depend on or affect external state, read or write files, or have other external effects. However, many effectful computations can be simulated in pure code (e.g. state can be passed between functions as a parameter), and Haskell uses a structure (or pattern) called a monad for convenient manipulation of effectful computations. In essence, a monad allows the creation of an environment where specific effects are made available. This can also include non-pure effects, and, unsurprisingly, I/O operations are only available in the appropriate monad. ${ }^{1}$

The type system distinguishes effectful computations from pure computations, and enforces that pure computations never can execute impure operations. For instance, I/O operations are guaranteed to only be executed in the context of the IO monad. A monad is a parametric type, so for some type a, the type IO a designates an I/O action which can be executed to produce a value of type a. For instance, get Char has type IO Char, as it is an I/O action that can produce a character. Apart from the ability to be executed by the run-time system, getchar is a normal value, and like other values it can be assigned to variables and manipulated with functions. Using combining functions, larger programs can be built that interact with their environment in complex ways.

In Haskell, STM is implemented as a monad, and transactions are confined to this environment. Similar to the IO example, a type STM a designates a transaction that, when executed, returns a value of some type a. In the STM monad, mutable data structures are available as explicitly declared transactional variables, or TVars. Using the same mechanism and syntax as other monads, simple transactions can be composed into more complex ones. Transactions can be executed in the IO monad, using the atomically function, which converts a value of type STM a to a value of type IO a.

It is important to note that TVars are only accessible from the STM monad. This makes them unavailable to non-transactional computations (i.e., plain functions), and the static type system rigidly enforces this encapsulation. Similarly, transactions have no means to modify other state, in particular, they are prevented from performing I/O operations or modifying global variables. This separation makes the

\footnotetext{
${ }^{1}$ While most monads can be - and usually are - implemented as simple libraries, the IO monad is special, and executed by the run-time system.
}

Haskell STM implementation safer to use, and may explain why STM implementations in other languages with less rigid type systems have been less successful.

\subsection{Genome Assembly and Scaffolding}

The sequencing process usually produces a large set of short fragments (or reads) from random positions in the genome. Given such a set of reads, the genome assembly problem is to reconstruct the originating genome sequence. The traditional approach is the method called overlap-layout-consensus (Bonfield et al., 1995; Myers et al., 2000), or OLC:

1. Identify overlaps by aligning each sequence against all others

2. Determine the layout - order and orientation - of the reads that is best supported by the alignments

3. Merge sequences according to layout to produce a single contiguous consensus sequence

The first step is trivially parallelizable (each read is independent of the others, and can be independently aligned), but the second step is more complicated. Usually, the problem is modeled as a graph where each read is a node, and there exists an edge between nodes if the corresponding reads are determined to overlap. Assembly is then equivalent to identifying a Hamiltonian path in the graph, which is an NPcomplete problem. $^{2}$

The layout phase processes the overlap graph to produce a linear progression of the reads, and although distant parts of the graph can be processed independently, care must here be taken if two operations attempt to modify the same nodes simultaneously. The implementation details of assemblers are not often published, but observation of some common OLC assemblers indicates that they commonly perform alignments in parallel, but later run the layout phase using a single thread of execution. ${ }^{3}$ This supports the view that constructing a correct locking scheme for doing graph updates in parallel is difficult. In addition, it would probably be inefficient, as it would incur locking overhead also for the noncolliding updates - likely to be the vast majority of them.

\footnotetext{
${ }^{2} \mathrm{~A}$ popular alternative to OLC is the de Bruijn assembly (Pevzner et al., 2001). This is less resource-intensive, as it avoids the all-against alignment phase, and it is equivalent to identifying an Eulerian path. But it is also easier to parallelize in practice, which may also be a factor that contributes to its popularity.

${ }^{3}$ E.g. Newbler only parallelizes computing alignments and generating output. (454 Life Sciences Corp., 2010)
} 
Genome scaffolding is closely related to assembly. Here, the assumption is that a genome has been sequenced and assembled into a set of contigs. In addition to overlaps, there exists external information about the orientation and order of the contigs. This is typically a set of paired reads, where the members of the read pairs are separated by some approximately known distance. As for assembly, it is not straightforward to implement a parallel scaffolding algorithm correctly using locking, and commonly used programs like SSPACE (Boetzer et al., 2011) are single-threaded.

Scaffolding simplifies the process in two ways: first, it reduces the amount of data that needs to be considered (E.g. for the sea louse assembly, the initial assembly involves up to one billion reads). Second, mapping reads to contigs make it practical to use standard alignment tools and file formats. For these reasons, the following will focus on the scaffolding problem.

\section{ALGORITHM AND IMPLEMENTATION}

A practical scaffolding program is likely to involve different heuristics to resolve ambiguous cases including repeats and chimeric contigs. As the purpose here is to demonstrate STM as an implementation technique, we implement a basic scaffolding algorithm that simply links together any pair of contigs that has a mutual best match, as described below. Matches are determined from aligned read pairs provided as a BAM (The SAM Format Specification Working Group, 2011) file.

First, the input BAM file is processed. By examining read pairs that map to the same contig, we obtain estimates for the expected distance between paired reads (called the insert length), and its variance. Also, the total number of contigs is extracted from the BAM file. Simultanously, the alignments relevant for scaffolding are extracted. In other words, each read of a pair must map near the ends of different contigs, and they must be oriented correctly. These alignments are stored in an associative data structure.

The scaffolding process uses two arrays, the contig array, which maps each contig to its scaffold, and the scaffold array, which for each scaffold stores the scaffold layout, i.e., the set of ordered and oriented contigs. Initially, each contig is in its own singleton scaffold.

The program now iterates over all contigs. For each contig $c$, the set of read pairs with one member matching near the 5' end of $c$ are extracted. The

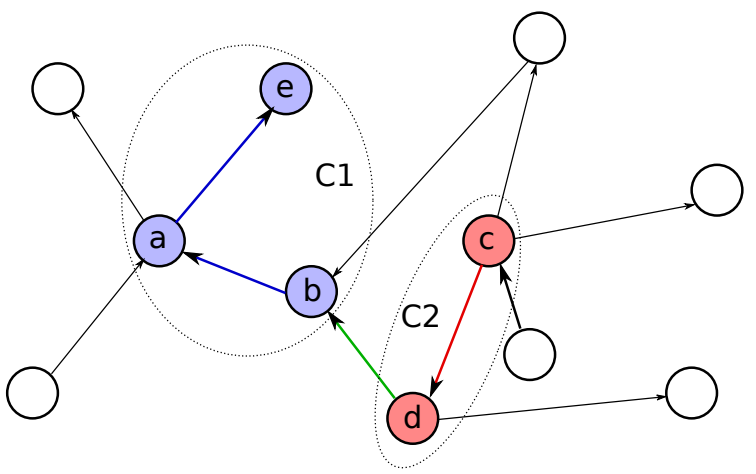

Figure 1: An example overlap graph. Two scaffolds are already identified, $\mathrm{C} 1$ (blue) containing nodes $\mathrm{a}, \mathrm{b}$, and e, and $\mathrm{C} 2$ (red) containing nodes $\mathrm{c}$ and $\mathrm{d}$. Adding the edge (green) from $\mathrm{d}$ to $\mathrm{b}$ will merge these into a single scaffold.

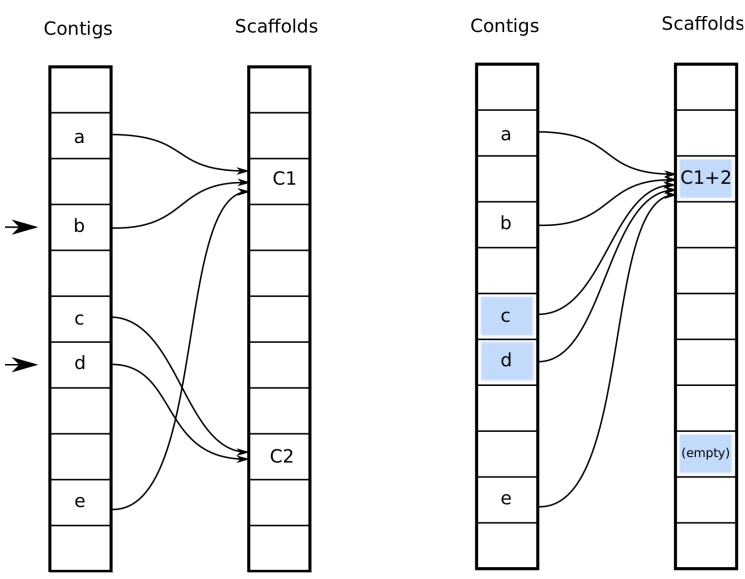

Figure 2: A schematic presentation of the arrays used in the scaffolding algorithm. As in Fig. 1, contigs $a, b$, and $e$ are initially (left) in scaffold $\mathrm{C} 1$, and contigs $\mathrm{C}$ and $\mathrm{d}$ are in scaffold $\mathrm{C} 2$. When the algorithm decides that contigs $b$ and $\mathrm{d}$ (indicated by arrows) should be adjacent, the scaffolds are merged, causing several cells to be updated (shaded, right).

contig $c_{l}$ to which the largest number of the mapped reads' mates map is identified. If this relationship is reciprocal (i.e, the reads pairs that map to $c_{l}$ have a majority of mates mapped to $c$ ), the contigs $c$ and $c_{l}$ are merged. The procedure is then applied similarly to the 3' end of $c$.

For instance, in the example graph in Figure 1, examination of node $b$ has determined that most mapped reads link it to $d$, and conversely, most reads mapping to $d$ link it back to b. This causes these two contigs to identified as adjacent, and their scaffolds are consequently merged.

Merging two scaffolds involves updating one scaffold's entry in the scaffold array to contain the new scaffold, and deleting the other scaffold's entry (see Figure 2). Then, the elements in the contig array cor- 
responding to contigs in the scaffold that was deleted are updated to point to the new scaffold.

To parallelize, we simply split the iteration of the contig array so that each thread iterates over an equally sized segment of the array. Note that even if threads work on separate array segments, they will affect contigs outside their segment.

Statistically, the merging operations will usually be independent if the arrays are large compared to the number of concurrent operations (threads). This also depends on the locality of merging criteria. The current implementation considers a subgraph consisting of three contigs at a time, but it could be extended to examine several candidates and links, in effect making the decision depend on a larger subset of the graph. This would increase the chance of colliding operations. In any case, collisions will occur occasionally, and a parallel implementation must take them into account.

STM here makes this process easy, and in fact, the code implementing this algorithm using mutable arrays in the IO monad and using transactions in the STM monad is exactly the same. Only the top-level function is different, as the STM version must spawn multiple threads that process an array segment each.

\section{RESULTS}

In order to test the implementation, the contigs resulting from the assembly of sea louse (Lepeophtheirus salmonis) sequences were used. This assembly was constructed using the Newbler program (Roche), which assembled approximately 50 million 454 reads (Margulies et al., 2005) into 292421 contigs.

As our pairing data, we use a set of 72200652 Illumina reads, where each pair consists of two $100 \mathrm{bp}$ reads, spaced about $150 \mathrm{bp}$ apart. The reads were aligned using BWA (Li and Durbin, 2009), resulting in 68569814 alignments (95\% of the reads), of these 10187580 alignments mapped the read and its mate to different contigs.

The program was compiled with GHC 7.0.2, using the -02 option. It was executed on a computer with eight Intel Xeon E7340 processors, using options + RTS -A100M. The parallel STM version was additionally compiled with -threaded, and run with -qg.

Figure 3 shows the running time for the scaffolding stage. We see that there is some overhead associated, both with using arrays of transactional variables (TArray) over regular mutable arrays (IOArray), and with running on the multi-threaded GHC run-time over the single-threaded one.

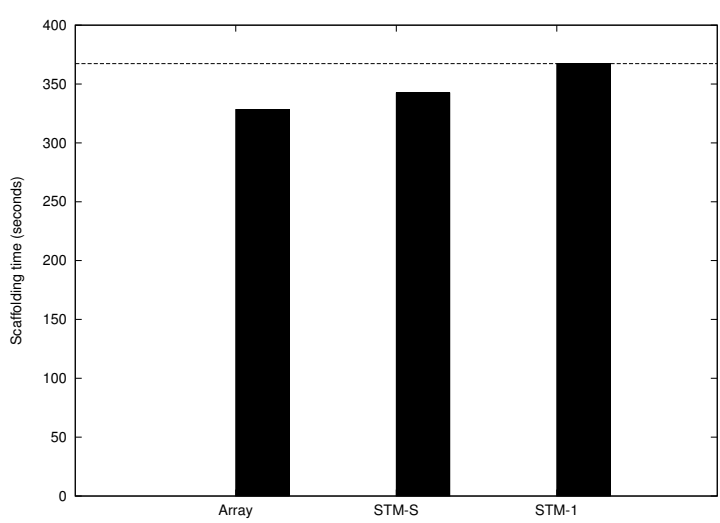

Figure 3: Speed spent in the scaffolding stage. "Array" is the implementation using mutable arrays, "STM-S" is the STM implementation running on the single-threaded runtime, and "STM-1" is the STM implementation using a single thread with the threaded run-time.

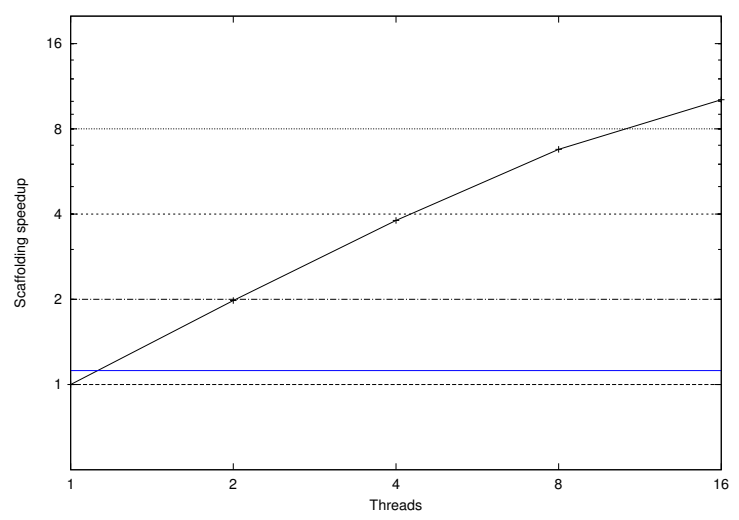

Figure 4: Speedup of the STM implementation with increasing number of threads. The blue line indicates the relative performance of the non-STM ("Array") implementation.

The STM implementation scales well. From Figure 4 , we see that as we increase the number of parallel threads, the speedup is close to the optimum, up to eight threads, matching the number of CPUs. The CPUs use hyperthreading, and each processor core appear to the OS as two processing units. Thus, the STM implementation is still achieving a substantial speedup going from 8 to 16 threads, even though it means running two threads per physical core.

The resulting scaffolds were checked against scaffolds produced by SSPACE, and were found to differ slightly, but for the most part, they identified the same layout of contigs. 


\section{DISCUSSION AND CONCLUSION}

Software transactional memory is most attractive when the program can be structured as set of mostlyindependent operations, and where each operation only involves a small set of variables. If the operations are completely independent, the problem most likely can be trivially partitioned, and if the number of variables involved in each operation is large, performance will deteriorate as the transaction log increases in size.

The overlap-layout-consensus approach to the sequence assembly problem fits well with these criteria, and is well suited to an STM approach. In the implementation presented, we observe a small overhead for using software transactional memory compared to regular arrays, and an additional overhead for using a multi-threaded implementation compared to a single threaded one, but the STM implementation scales well with the number of threads, and already with two threads it is substantially faster. Although the results here are very promising, it remains to be seen how far they generalize, both as the number of CPUs increase, and to variations of the algorithm.

This analysis has concentrated on how to improve the run-time performance of the scaffolding process. This is an important goal in itself, but it is even more important to improve the quality of the resulting genome assembly.

The composability of STM lets the programmer easily refactor the program or otherwise modify the algorithm without introducing deadlocks or other synchronization problems. For instance, the current implementation only considers the potential nearest neighbors of each contig. Extending it to take into account a larger subgraph is one possibility in improving the result. With a traditional locking scheme, this would likely increase the complexity substantially. With STM, it would at worst increase the chance of collisions between transactions, leading to more retries, and consequently a slightly slower program.

The source code for the implementation is available $^{4}$ under the General Public License.

\section{REFERENCES}

454 Life Sciences Corp. (2010). 454 Sequencing System Software Manual, v 2.5p1, part C. 454 Life Sciences Corp., Branford, CT 06405.

\footnotetext{
${ }^{4} \mathrm{http} / / /$ malde.org/ ketil/biohaskell/stmasm
}

Boetzer, M., Henkel, C. V., Jansen, H. J., Butler, D., and Pirovano, W. (2011). Scaffolding pre-assembled contigs using SSPACE. Bioinformatics, 27:578-579.

Bonfield, J. K., Smith, K. F., and Staden, R. (1995). A new DNA sequence assembly program. Nucleic Acids Research, 23:4992-4999.

Brevnov, E., Dolgov, Y., Kuznetsov, B., Yershov, D., Shakin, V., Chen, D.-Y., Menon, V., and Srinivas, S. (2008). Practical experiences with java software transactional memory. In Proceedings of the 13th ACM SIGPLAN Symposium on Principles and practice of parallel programming, PPoPP '08, pages 287-288, New York, NY, USA. ACM.

Harris, T., Marlow, S., Peyton-Jones, S., and Herlihy, M. (2005). Composable memory transactions. In Proceedings of the tenth ACM SIGPLAN symposium on Principles and practice of parallel programming, PPoPP '05, pages 48-60, New York, NY, USA. ACM.

Lee, E. A. (2006). The problem with threads. Technical Report UCB/EECS-2006-1, EECS Department, University of California, Berkeley. The published version of this paper is in IEEE Computer 39(5):33-42, May 2006.

Li, H. and Durbin, R. (2009). Fast and accurate short read alignment with burrows-wheeler transform. Bioinformatics, 25:1754-1760.

Margulies, M., Egholm, M., Altman, W. E., Attiya, S., Bader, J. S., et al. (2005). Genome sequencing in microfabricated high-density picolitre reactors. Nature, 437:376-380.

Myers, E. W., Sutton, G. G., Delcher, A. L., Dew, I. M., Fasulo, D. P., Flanigan, M. J., et al. (2000). A whole-genome assembly of drosophila. Science, 287(5461):2196-2204.

Ni, Y., Welc, A., Adl-Tabatabai, A.-R., Bach, M., Berkowits, S., Cownie, J., Geva, R., Kozhukow, S., Narayanaswamy, R., Olivier, J., Preis, S., Saha, B., Tal, A., and Tian, X. (2008). Design and implementation of transactional constructs for $\mathrm{c} / \mathrm{c}++$. In Proceedings of the 23rd ACM SIGPLAN conference on Objectoriented programming systems languages and applications, OOPSLA '08, pages 195-212, New York, NY, USA. ACM.

Pevzner, P. A., Tang, H., and Waterman, M. S. (2001). An eulerian path approach to dna fragment assembly. Proceedings of the National Academy of Sciences, 98(17):9748-9753.

Shavit, N. and Touitou, D. (1995). Software transactional memory. In Proceedings of the fourteenth annual ACM symposium on Principles of distributed computing, PODC '95, pages 204-213, New York, NY, USA. ACM.

The SAM Format Specification Working Group (2011). The SAM Format Specification.

\section{APPENDIX}

The code for the merging operation (as illustrated in Figure 2 is given below. Note that the type signature is 
not given, and the code will typecheck and run without modification in either the IO monad or the STM monad. In STM, each array cell is a TVar, and the merge operation must be part of a transaction. If another thread modifies any of the involved array locations before the transaction completes (say by merging one of the clusters with a different cluster), the transaction will be aborted and restarted. In IO, there are no such guarantees, and the function can only be run safely in a single thread.

The function takes as input parameters arrays of contigs (each pointing to a cluster) and scaffolds (echo containing the list of its elements), and a pair of contigs. It then merges the scaffolds that contain the given each contig from the pair.

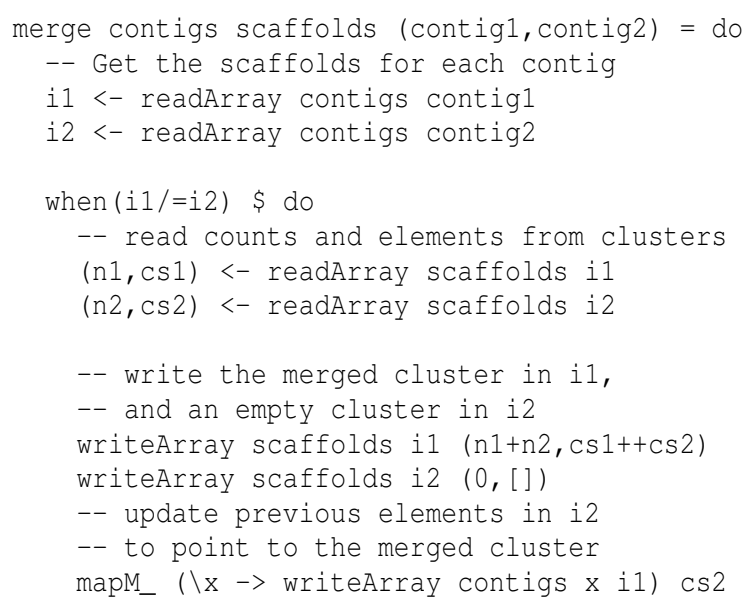

